\title{
Broadrick Occlusal Plane Analyzer
}

\author{
Sangeetha Chokalingam ${ }^{1}$, Dr.Gururaj Rao ${ }^{2}$ \\ ${ }^{I}$ (Undergraduate Student, Saveetha Dental College and Hospitals, India) \\ ${ }_{2}^{2}$ Senior Lecturer, Department of Oral and Maxillofacial Prosthodontics, Saveetha Dental College and \\ Hospitals, India)
}

\begin{abstract}
Full mouth rehabilitation cases require meticulous treatment planning. Such instrument that often is used to achieve an occlusal equilibrium for full mouth rehabilitation cases is the Broadrick Occlusal Plane Analyzer. Also known as BOPA, this is instrument has only been adapted to a few articular system which limits its use. The review paper highlights the parts, the preparation and the use of Broadrick Occlusal Plane Analyzer.
\end{abstract}

Keywords: BOPA, Broadrick Occlusal Plane Analyzer,Full Mouth Rehabilitation, Occlusal Plane, Articular System

\section{Introduction}

In prosthodontics or rather in fixed prosthodontia, when it has been analyzed that restoration or reconstruction of most or all of the posterior teeth is needed, the instrument, Broadrick's Occlusal Plane Analyzer is often the easiest and hassle free instrument that is used ${ }^{[1]}$.BOPA is used to determine and achieve an occlusal plane that fulfills both the functional, occlusal as well as the aesthetics requirement in cases that require full mouth rehabilitation ${ }^{[1]}$.Most of the teeth that are present, are usually attrited or fractured and have no occlusal or functional equilibrium ${ }^{[1]}$. The term 'plane' is used in association with flat surfaces ${ }^{[1]}$. In case of the occlusal plane, instead of flat surfaces, it represents the average curvature of the occlusal surfaces. ${ }^{[1]}$

The term 'plane' is better explained in the glossary of prosthodontic terms ${ }^{[2]}$.It defines the occlusal plane as 'the average plane established by the incisal and occlusal surfaces of the teeth ${ }^{,[2]}$.The occlusal plane has its own benefits, both anteriorly and posteriorly. It helps in the mastication,posteriorly and in the anterior region it helps in the phonetics and aesthetics ${ }^{[2]}$.Functionally, one of the key factors that governs the occlusal balance is the inclination of the occlusal plane ${ }^{[2]}$. Any changes, even the slightest bit in the plane of occlusion will modify both the physical and functional relationship of the oral musculature, which will result in an alteration in the comfort, function as well as the aesthetic value ${ }^{[2]}$.

\section{Determination Of Plane Of Occlusion}

The plane of occlusion is represented by the average curvature of the occlusal surfaces instead of the flat surfaces ${ }^{[1]}$. The position of the anterior teeth is usually the determinant for esthetic consideration, which is of outmost importance for phonetics and anterior guidance ${ }^{[1]}$. There are two curves that determine the position of the posterior teet ${ }^{[1]}$.These curves are Curve of Spee which is the anteroposterior curve and Curve of Wilson, which the mediolateral curve ${ }^{[1]}$. It was based on an anthropological study in 1919, that Monsoon proposed the anteroposterior curve of the teeth forms a sphere, with the center of rotation located in the region of the glabella ${ }^{[1]}$. The estimated radius of the sphere is said to be 4 inches ${ }^{[1]}$.

There are selective ways which are used to determine an acceptable plane of occlusion with the presence of the natural dentition ${ }^{[1]}$. Out of which, there are three ways in which the plane of occlusion can be achieved. These methods are of direct analysis of the natural teeth which is through selective grinding and indirect analysis which is on the face-bow mounted casts with properly set condylar paths ${ }^{[1]}$. There is also another indirect analysis which is used when the restoration and rehabilitation of most or all posterior teeth is needed $^{[1]}$. This method is known as the Pankey-Mann-Schuyler(PMS) method with the Broadrick occlusal plane analyzer ${ }^{[1]}$. The advantage of this method is that it is a practical and easy method in determining the initial occlusal plane on the casts ${ }^{[1]}$. It is also helpful in locating the cusp tips of the posterior teeth as well as serves as a guide for the amount of tooth reduction that needs to be done or is needed for the intended occlusal planes ${ }^{[1]}$.

For the initial development of the mandibular occlusal plane in the casts as well as in the later stages for the contours of the definitive restorations and for guidelines in the actual tooth preparation ${ }^{[3]}$. Since the Curve of Spee maybe altered in cases such as extrusion of teeth, rotation and tipping, the reconstruction of the dentition to such an altered occlusal plane can cause interference in the posterior region ${ }^{[3]}$.Such protrusive interference in the posterior region could possibly cause abnormal activity in the muscles, especially the masseter and the temporalis muscle and in the temporomandibular joint ${ }^{[3]}$. Reconstructing the Curve of Spee to pass through the 
mandibular condyle, which causes posterior disocclusion on mandibular protrusion could prevent the abnormal muscle activity ${ }^{[3]}$.

\section{Broadrick Occlusal Plane Analyzer}

The Broadrick Flag allows the construction of the Curve of Spee in perfect harmony with the anterior condylar guidance allowing total posterior tooth disclusion on mandibular protrusion ${ }^{[3]}$. This results in the aesthetic positioning and proper functioning of the mandibular incisors ${ }^{[3]}$.

The Broadrick occlusal plane analyzer(BOPA) has only been adapted to a few articular system as it is an expensive instrument which limits its use universally ${ }^{[1],[4]}$. Some of the articular system that uses Broadrick occlusal plane analyzer is the Denar Anamark Fossae and all models of the Hanau articulators ${ }^{[1]}$. As not many articular systems support the flag and also for the manufactures of the semi-adjustable articulators who do not provide with such flag, a custom made Broadrick flag could be used ${ }^{[1]}$. The flag could be constructed with the use of clear acrylic resin with several modifications to the semi-adjustable articulator ${ }^{[1]}$. The Broadrick Occlusal Plane Analyzer consist of a(Fig.1):-

1- Card Index.

2- Bow Compass with graphite leads with an extra center point and needle point.

3- Scribing knife.

4- Plastic Record cards.

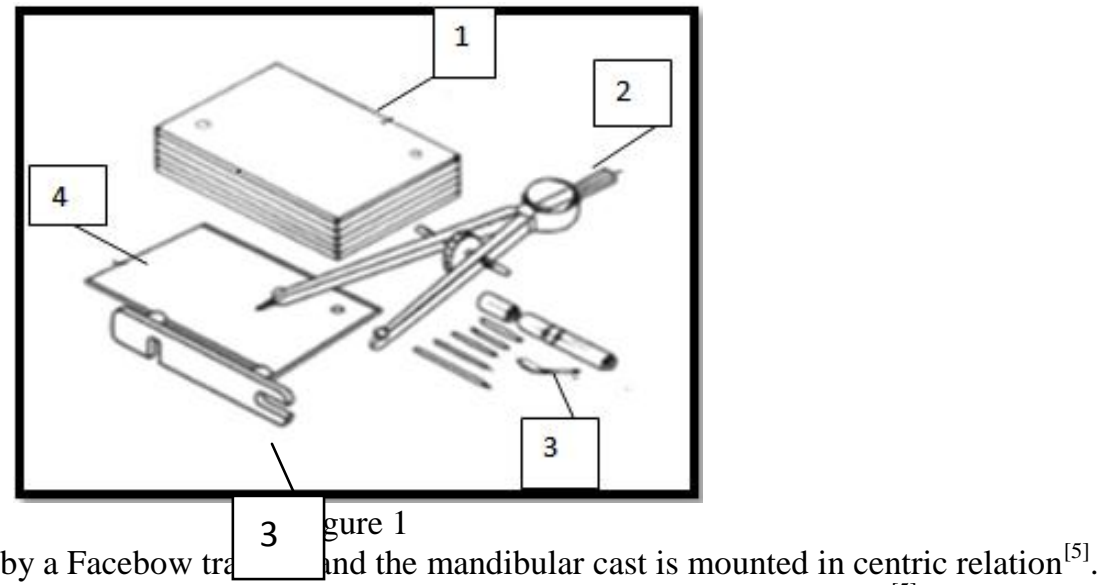

The maxillary cast is mounted by a Facebow tre 3 ind the mandibular cast is mounted in centric relation ${ }^{[5]}$. An accessory Split-Cast Mounting plate is mounted on the Upper member of the articulator ${ }^{[5]}$. This split cast allows rapid cast removal and accurate replacement during the survey ${ }^{[5]}$. It also provides a visual guide for adjustment of the articulator to protrusive and lateral interocclusion records ${ }^{[5]}$.

\section{Preparation Of The Analyzer}

1- The card index is placed on the upper member with the open end around the incisal pin and the slot on the side around the mounting plate thumbscrew ${ }^{[5]}$. (Fig. 2)

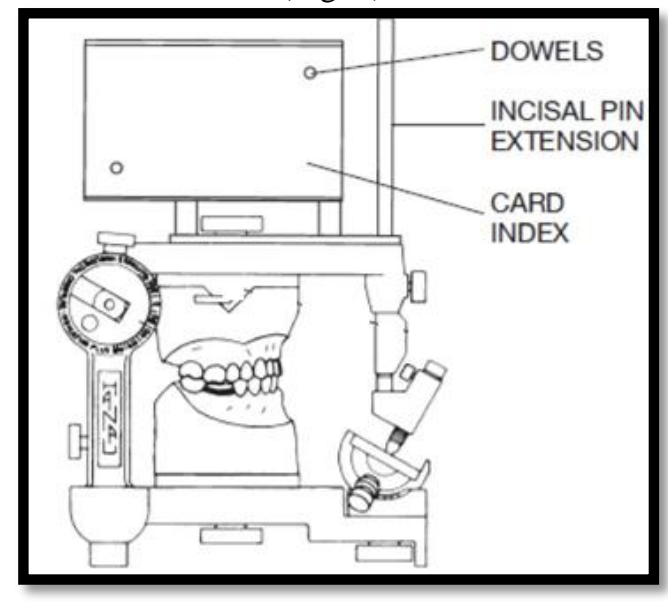

figure 2

2- The thumbscrew is tighten to hold the card index in position ${ }^{[5]}$.

3- If there is an Orbitale indicator mounted on to the articulator, care should taken to remove it in order to mount the card index ${ }^{[5]}$. 
4- Press a plastic record card over the dowels on the right side of the card index ${ }^{[5]}$. The cards are matte finished on both sides, thus accepts ink or pencil markings readily ${ }^{[5]}$.

5- A relatively small divergence between arcs of 3-3/4 ", 4" and 5" radii over the functional occlusal surfaces on the lower posterior teeth ${ }^{[5]}$. The radius of sphere in the Curve of Spee is suggested to be at 3.75 inches in skeletal Class II relationship, whereby a 5 inch radius is more appropriate in a skeletal Class III relationship ${ }^{[3]}$. A 4 inch radius is considered normal and most often used in majority of cases especially in Class I relationships. (Fig. 3)

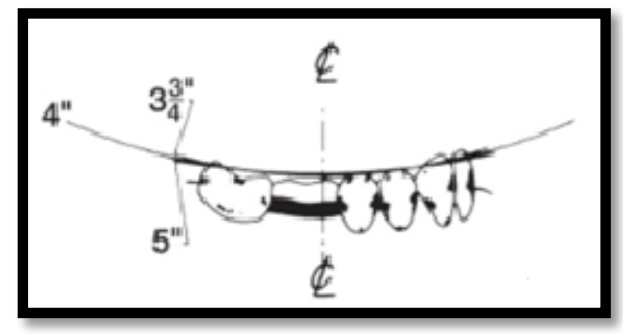

figure 3

6- Then tapered pin is withdrawn from the split mount cast plate and the upper cast is removed ${ }^{[5]}$. A lead piece is inserted on to the compass and it is adjusted to a selected radius ${ }^{[5]}$.

7- The center point of the compass is adjusted to the anterior survey point (A.S.P), which is usually the distoincisal of the canines ${ }^{[5]}$. If it is noticed that the cusp of the canine is attrited or worn our flat, the anterior survey point may be at the incisal edge ${ }^{[5]}$. If a point is selected as the most desirable point that 'beams' the line and plane of occlusion posteriorly, it is marked on to the canine and not changed ${ }^{[5]}$. ( Fig.4)

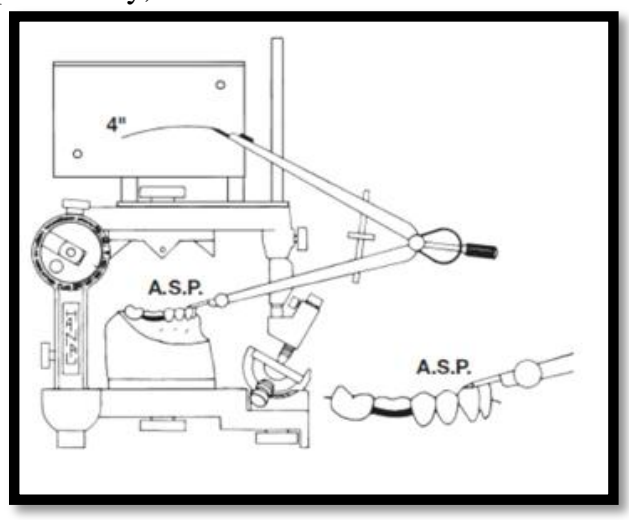

figure 4

8- $\quad$ As the center of the compass is positioned on the A.S.P, a long arc of 3 inches is applied on to the plastic record card ${ }^{[5]}$. The occlusal plane survey center (O.P.S.C) will eventually be located on some point on the $\operatorname{arc}^{[5]}$.

9- A posterior most point (P.S.P) is selected at the distobuccal cusp of the lower last molar ${ }^{[5]}$.(Fig.5)

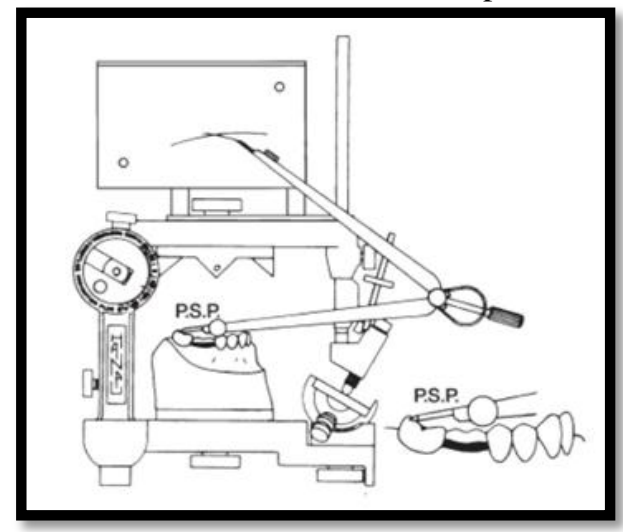

figure 5

10- In such cases where there is an absence of the lower molar, the upper cast is replaced and a soft modeling compound is placed over the ridge, closing the articulator until the incisal pin contacts the incisal guide in a centric relation ${ }^{[5]}$. The compound is chilled and the excess is carved away, leaving only compound contacting into the upper fossae, simulating the lower buccal cusp ${ }^{[5]}$. 
11- The upper cast is removed and a posterior survey point (P.S.P) is selected on the modeling compound ${ }^{[5]}$.

12- The center point of the compass is positioned on the P.S.P and an arc is applied to intersect the arc from the A.S.P. $P^{[5]}$.

13- Alternate to the molar P.S.P, is a position on the condylar element of the articulator, at its anterior intersection with the condylar shaft ${ }^{[5]}$.

14- The center point of the compass is positioned on the condylar posterior survey point (C.P.S.P) and an arc to intersect the arc formed from A.S.P ${ }^{[5]}$.

15- The needle point is then swept over the occlusal surfaces of the lower posterior teeth to see how the arc conforms to the existing occlusal plane ${ }^{[5]}$. This occlusal plane survey center ( O.P.S.C) is shifted on the long arc on the plastic record card, the A.S.P line, until the most acceptable plane of occlusion and line is found $^{[5]}$.

16- If there is a need to raise the line and plane of occlusion at the distal end, the center point is moved anterior to the arc intersection ${ }^{[5]}$. To lower the line and plane of occlusion, the point is moved posterior of the intersection.

17- After repeated trial and retrial, the ideal survey center forming the most acceptable line and plane of occlusion will be located ${ }^{[5]}$.(Fig.6)

18- The scribing knife is for the placement into the compass for scribing and cutting the plaster, compound or wax during the occlusal plane correction ${ }^{[5]}$.

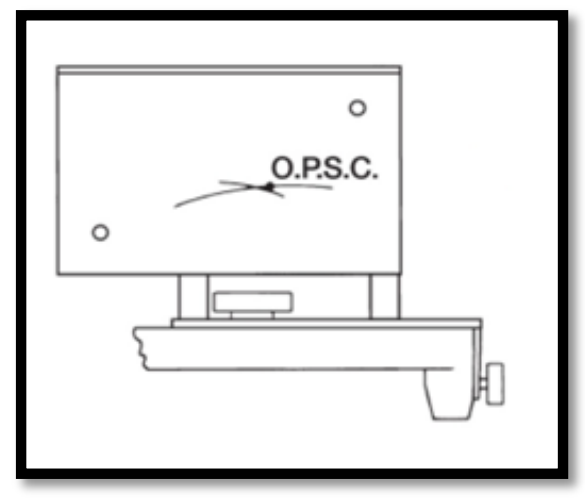

figure 6

\section{Discussion}

There are also a number of instruments which has been proposed over the time for orientation of the occlusal plane. Back in 1952, JE Scott described an instrument called 'the bite plane leveler' which consist of two flat, horizontally curved, parallel bars ${ }^{[2]}$. The downside of this instrument is that it only be used to check the parallelism of occlusal plane to the ala-targus line ${ }^{[2]}$. The use of J-plane' with the use of a fox plane to establish the occlusal plane orientation was suggested by Nikzad s Javid in $1974^{[2]}$. This instrument was meant to be used instead of a metallic scale ${ }^{[2]}$. Kazanoglu and John W Ugner in 1992 explained that 'Camper's plane indicator' for the determination of the occlusal plane orientation. It consist of two metal plates connected with a long aluminium $\operatorname{rod}^{[2]}$. The disadvantage of this instrument is that it is time consuming as the orientation with the interpupillary line and the right and left Camper's plane is done separately ${ }^{[2]}$. In 1998 Urbano, Santana-Penim and Maria J Mora suggested another device for determining the inclination of the occlusal plane ${ }^{[2]}$. It was described that the device, which is made up of stainless steel, ' $U$ ' shaped with one shorter inner arm designed to be positioned against the occlusal surface and another longer,outer arm which lies outside the mouth[2].

\section{CONCLUSION}

The usage of this instrument offers great help in terms of esthetics and masticatory functions through the proper orientation of the occlusal plane ${ }^{[1]}$. As the radius of sphere in Curve of Spee varies between patients, Broderick occlusal plane analyzer is often the instrument used ${ }^{[3]}$. The radius of sphere in the Curve of Spee is suggested to be at 3.75 inches in a Class II skeletal relationship, whereby a 5 inch radius is more appropriate in a skeletal Class III relationship, as anything less would create a steep posterior curve causing further posterior interference $^{[3]}$. For a more consistent approach, the author suggest the use of the condylar element as the posterior survey point (P.S.P) in the likehood of a tilted, attrited or mesially drifted molars.( Fig.7) The simple modification procedures enables the practitioners to use an occlusal plane analyzer as an important diagnostic tool with a widely used semi-adjustable articulator ${ }^{[1]}$. 


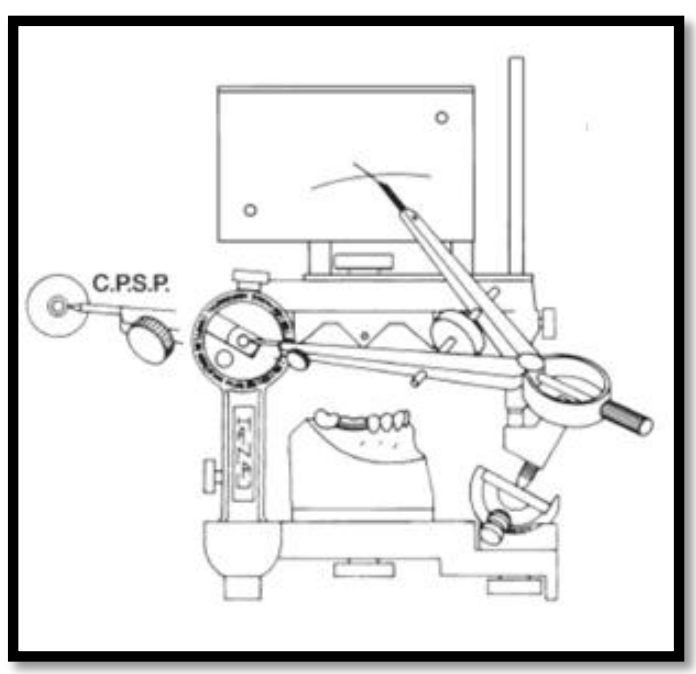

figure 7

\section{References}

[1] S.V. Bedia, S.P. Dange and A.N. Khalikar, Determination of the occlusal plane using a custom-made occlusal plane analyzer: A clinical report, Journal of Prosthetic Dentistry, 98(5), 2007,348-352.

[2] R.Gupta, Occlusal Plane Analyzer: A customized Device for Determining the Occlusal plane, International Journal of Prosthodontics and Restorative Dentistry, 1(2), 2011,97-100.

[3] S.Manvi, S.Miglani, C.L. Rajeswari, G. Srivatsa and S. Arora, Occlusal Plane Determination using Custom Made Broadrick Occlusal Plane Analyser: A case control study, International Scholarly Research Network Dentistry, 2012,373870

[4] S.Chaturvedi, A.K. Verma, M.Ali, M.Shah, Full Mouth Rehabilitation using a custom-made broadrick flag: A case report, International Journal of Case reports and images, 3(5), 2012, 41-44

[5] W.M Corporation, Restorative oral health(U.S.A, Whip-Mix Corporation-West,2008) 